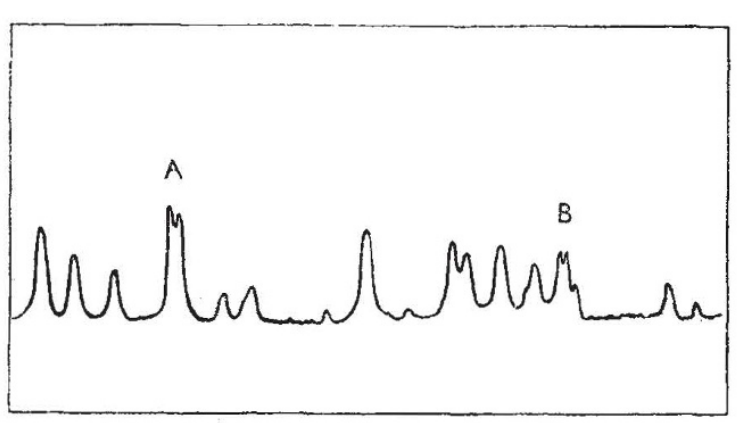

Frequency increasing $\longrightarrow \longrightarrow$

Fig. 2

spectrometer with thermocouple detector. It seems probable that in the future the limit of resolving power in the near infra-red will be of the order of $0.05 \mathrm{~cm} .^{-1}$, compared to a pre-war limit of $0.5 \mathrm{~cm}^{-1}$.

We would also emphasize the great advantages to be derived from the exceptional speed of response of the lead sulphide detector, which has a time constant of considerably less than 0.001 sec. compared to the Schwarz thermocouple of about 0.1 sec. and the thermistor bolometer of about 0.01 sec. Quite apart from the increased speed with which spectra can be plotted, this short time-constant allows one to chop the radiation at a high frequency (we actually used 800 c. $/$ sec.) and employ a tuned detector system which entirely eliminates drift. Furthermore, the use of a lead sulphide cell in connexion with our cathode ray presentation of spectra ${ }^{4}$ will allow much higher scanning speeds than can be achieved with a bolometer. This means that a more truly instantaneous picture of the spectrum can be obtained, or alternatively a wider range of the spectrum can be viewed continuously, than with a bolometer.

A full description of these results will be published shortly elsewhere.

$\mathrm{We}$ wish to acknowledge our indebtedness to the Admiralty for the loan of the cells used, and to the Telecommunications Research Establishment at Malvern for the loan of the amplifier equipment.

G. B. B. M. SuTherLland

D. E. Blackwell

P. B. Fellatet

Laboratory of Colloid Science, Cambridge.

Oet. 31 .

${ }^{1}$ Starkiewicz, J., Sosnowski, L., and Simpson, O.. Nature, 158, 28 (1946). Lee, E., and Parker, R. C., Nature, 158, 518 (1946). Cashman, R. J., J. Opt. Soc. Amer., 36, 356 (1946).

3. Nielsen, H. H., Phys. Rev., 62, 422 (1942).

'Daly, E. F., and Sutherland, G. B. B. M., Nature, 157, 547 (1946).

\section{Angular Momentum of the Solar System}

ONE of the main difficulties to be met by any theory about the origin of the solar system is the difficulty of accounting for the present distribution of its angular momentum.

This difficulty can be stated in the following way. If the material of the planets has originated from the sun, it is difficult to understand why the average angular momentum per unit mass of the planetary material should be about 50,000 times larger than the average angular momentum per unit mass of the solar material. If, however, the planetary material was at the beginning present in a nebula around the sun, the density of this nebula was too small for a condensation into solid bodies under the sole in. fluence of the gravitational forces (Roche). If, finally, the condition from which the evolution started was such that the material of the sun and the planets together was smoothed out in a nebula, the total angular momentum of the system was far larger than at present, in contradiction with the law of the conservation of angular momentum.

The apparent failure of the theories of Kant and Laplace to explain this point has led to several 'catastrophic' theories being put forward. Von Weizsäcker ${ }^{1}$ gives in his recent theory, which is along the lines originally proposed by Kant and Laplace, an explanation which is, however, not completely convincing.

It seems, however, that it may well be possible to account for the present distribution of the angular momentum, without introducing interactions with foreign bodies, if we take into account that the condensation into planets will probably not take place under the influence of the gravitation alone, but will be a consequence of a process of condensation similar to that occurring in a supersaturated vapour, or to the process of the formation of smoke particles in interstellar space, as has been remarked already by Lindblad ${ }^{2}$ and Jeffreys ${ }^{3}$.

We may start thus from a situation where the sun is surrounded by a rotating nebula possessing an angular momentum which is about equal to the total angular momentum of the planetary system at present. The fact that the density in this nebula will be too low to allow condensation by gravitational action no longer prevents the actual condensation, since this condensation can proceed in the way mentioned above.

Institute for Theoretical Physics,

D. TER HAAR

University of Copenhagen,

Denmark.

Nov. 4.

1 Weizsäcker, C. F. von, Z. Astrophys., 22, 319 (1944).

2 Lindblad, L., Nature, 135, 133 (1935).

S Jeffreys, H. Nature, 153, 140 (1944).

\section{Physical Basis of a New Theory of Absorption of Ultrasonics in Liquids}

The relaxation theory of Kneser ${ }^{1}$, the diffusion theory of Lucas ${ }^{2}$, and other theories ${ }^{3}$ have not satisfactorily explained the excessive absorption of ultrasonics in liquids, unaccompanied by any sensible dispersion. In the following we offer a possible mechanism for the phenomenon.

The relevant difference in the structure of gases and liquids is that in the former the rupturing tendeney indicated by $k T$ is much more than the cohesive tendency of intermolecular attraction forces, whereas in liquids the reverse is the case. In gases we picture the molecules as free except at collisions, on which occasion they rearrange their energies in different degrees of freedom. But the distances to be reached in collisions in gases are already nearly reached in liquids at all times, and so the conditions arising at collisions in gases lose their force in explaining the characteristic phenomena in liquids, for these conditions were present all the time. The existence of this fundamental difference becomes evident on examining the opposite temperature variation of compressibility and viscosity in gases and in liquids. In a liquid and in the ordinary state, the electron atmospheres of the molecules are affecting each other at the close 